\title{
Medical thoracoscopy and gastroscopy for the treatment of intrathoracic anastomotic leakage following esophagectomy
}

\author{
XIAO-HAI LI ${ }^{1,2^{*}}$, YI HU ${ }^{1 *}$, TIE-HUA RONG ${ }^{1}$, XIAO-DONG LI ${ }^{1}$, XIAO-DONG SU ${ }^{1}$, \\ HONG YANG $^{1}$, AMOS ELA BELLA ${ }^{1}$ and JIAN-HUA FU ${ }^{1}$ \\ ${ }^{1}$ Department of Thoracic Surgery, Sun Yat-sen University Cancer Center, State Key Laboratory of Oncology \\ in Southern China, Guangzhou, Guangdong 510060; ${ }^{2}$ Department of Cardiothoracic Surgery, \\ The First Affiliated Hospital of Guangzhou University of Traditional Chinese Medicine, \\ Guangzhou, Guangdong 510405, P.R. China
}

Received March 21, 2012; Accepted June 29, 2012

DOI: $10.3892 / \mathrm{ol} .2012 .957$

\begin{abstract}
Intrathoracic anastomotic leakage following esophagectomy is extremely difficult to manage appropriately. The outcomes of conservative management strategies are often disappointing, particularly in patients who develop adhesions of the pleural cavity and multiloculated empyema. This study describes a novel approach using combined thoracoscopy and gastroscopy in two cases. Thoracoscopy under local anesthesia was used to dissect the septations within the multiloculated empyema and remove the infected focus by direct visualization, and gastroscopy was subsequently performed to place a nasogastric or sump tube around the leak. The outcomes of both procedures were satisfactory: the empyemas almost completely resolved, the anastomotic leak closed quickly and there was adequate lung re-expansion. Accordingly, the combination of thoracoscopy and gastroscopy for the treatment of intrathoracic anastomotic leak post-esophagectomy may be an effective, safe, minimally-invasive, simple and inexpensive procedure.
\end{abstract}

\section{Introduction}

Intrathoracic anastomotic leakage is an extremely intractable complication that occurs following esophagectomy, with an incidence that ranges from 1.3 to $5.1 \%$ (1-3). The mortality rate is $12-46.2 \%$ (1-3). In general, the effect of conservative treatments, including conventional chest tube drainage, nasogastric decompression and nutritional support, is often poor, particularly in patients with adhesions of the pleural cavity

Correspondence to: Dr Jian-Hua Fu, Department of Thoracic Surgery, Sun Yat-sen University Cancer Center, State Key Laboratory of Oncology in Southern China, Guangzhou, Guangdong 510060, P.R. China

E-mail: fujh@sysucc.org.cn

"Contributed equally

Key words: intrathoracic anastomotic leak, thoracoscopy, gastroscopy and multiloculated empyema (1-4). This study describes the combined application of medical thoracoscopy followed by gastroscopy for the treatment of an intrathoracic anastomotic leak in two patients. The study was approved by the IRB of Sun Yat-sen University Cancer Center. Consent was obtained from both of the patients.

\section{Case reports}

Case 1. A 58-year-old male patient with middle-third thoracic esophageal squamous carcinoma was confirmed to have an intrathoracic anastomotic leak 10 days after esophagectomy and a right-sided contained empyema 4 weeks after esophagectomy. The condition of the patient did not improve following jejunostomy, conventional chest tube drainage and gastrointestinal decompression for 8 weeks. Gastroscopy (Fig. 1A) confirmed that a large intrathoracic anastomotic leak existed in the posterior right-sided wall of the esophagus. Two nasogastric tubes were then respectively placed into the residual stomach and the sump around the leak. A computed tomography scan was performed prior to thoracoscopy in order to select the incision accurately to facilitate exploration, debridement and drainage. Thoracoscopy was then performed under continuous sedation and local anesthesia. The port was inserted through a trocar via an incision on the right mid-clavicular line, between the second and third intercostal spaces. These adhesions and septations were dissected with biopsy forceps and electrocautery. After removing the infected focus, the anastomotic leak could be observed at the upper pole of the empyema cavity with some refluxed stomach content. The lateral side of the nasogastric tube entering the sump around the leak could also be visualized through the anastomotic leak (Fig. 2). After the infected focus and digestive secretions around the anastomotic leak were completely removed, a chest tube was placed into the sump around the anastomotic leak (Fig. 3). The correct orientation of the chest tube was attained by slowly adjusting the head direction of the flexible thoracoscope and the chest tube replaced a cytology brush catheter via the thoracoscope. Postoperatively, the patient continued to receive conservative treatments and chest lavage. A barium and chest radiography confirmed the closure 

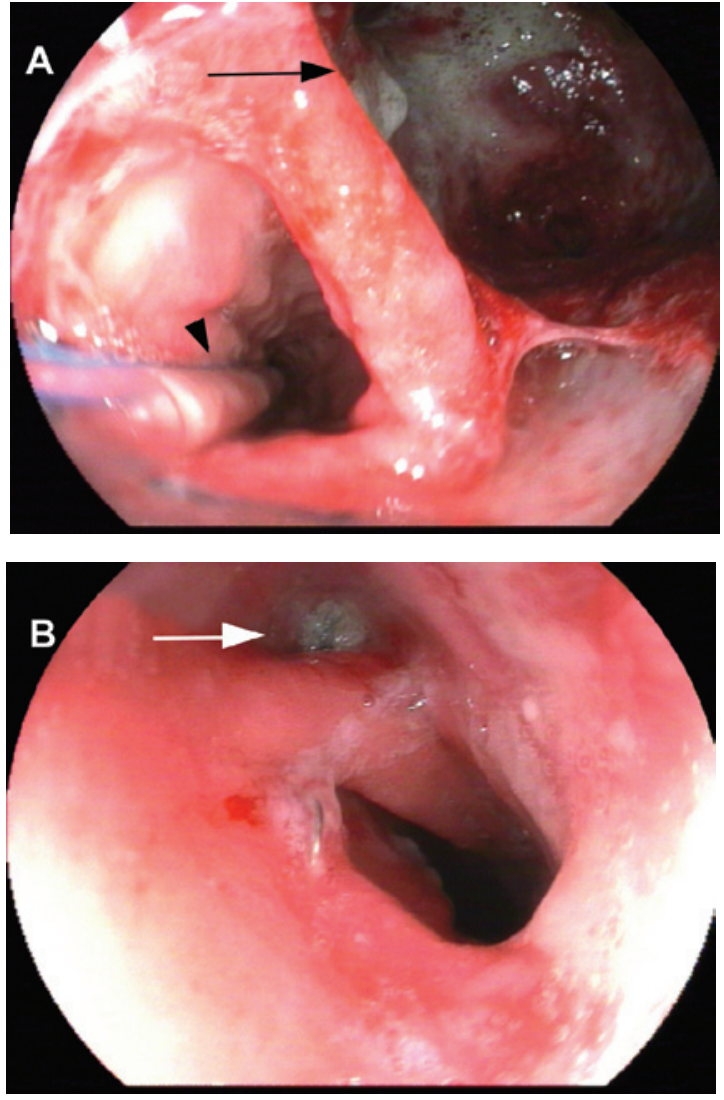

Figure 1. (A) Gastroscopy (Case 1) revealed an intrathoracic anastomotic leak (arrow) and the residual stomach (arrowheads). (B) Gastroscopy revealed closure of the anastomotic leak and a small residual sump (arrow) 5 months after the combined interventions.

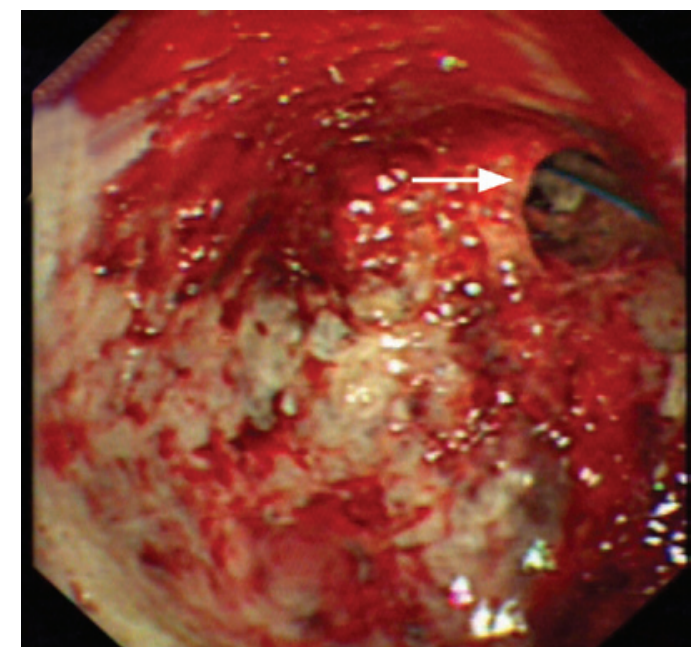

Figure 2. Thoracoscopy (Case 1) revealed the anastomotic leak and the lateral aspect of the nasogastric tube entering the sump around the leak (arrow).

of the anastomotic leak and right lung re-expansion 3 months after the combined procedure. Gastroscopy revealed closure of the anastomotic leak and a small residual sump (Fig. 1B) 5 months after the combined procedure.

Case 2. A 50-year-old male patient with middle-third thoracic esophageal squamous carcinoma was confirmed to have an

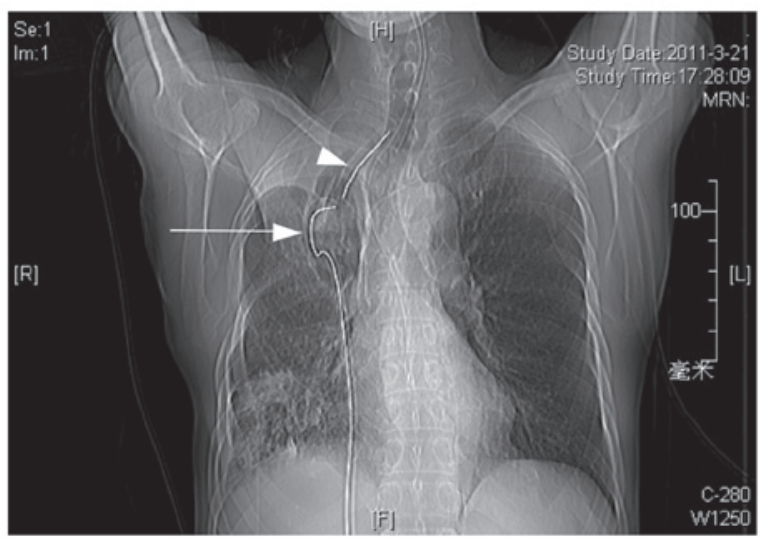

Figure 3. Chest radiography (Case 1) demonstrated the position of the chest drainage tube (arrow) and the nasogastric tube (arrowheads) in the sump around the anastomotic leak.

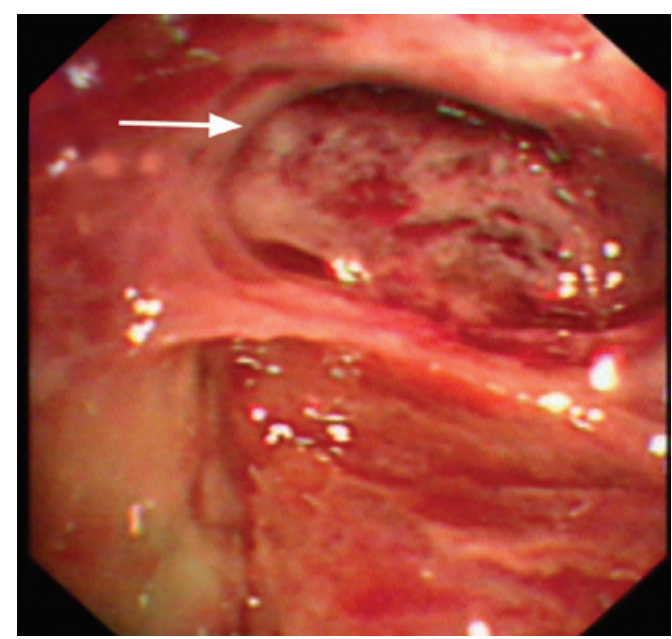

Figure 4. The sump around the anastomotic leak (arrow) could be observed after the infected focus was removed under thoracoscopy (Case 2).

intrathoracic anastomotic leak and a right-sided contained empyema 13 days after esophagectomy. Therefore, the combined application of thoracoscopy and gastroscopy was performed, as described in Case 1. The trocar was inserted through an incision on the right parasternal line between the third and fourth intercostal spaces. The purulent pleural effusion and fibrinopurulent focus were completely removed under direct vision. The sump around the anastomotic leak could then be observed at the superiolateral pole of the empyema cavity (Fig. 4). A chest tube was placed at the lowest aspect of the empyema cavity. Pleural cavity lavage was then performed through the chest tube. The condition of the patient improved 2 weeks after the combined procedure.

\section{Discussion}

Intrathoracic anastomotic leaks may easily cause multiloculated and contained empyemas. In general, the effect of conservative treatments, including conventional chest tube drainage, nasogastric decompression and nutritional support, is poor in such patients (1-4). This study describes 
two patients with an anastomotic leak post-esophagectomy, who underwent medical thoracoscopy. The septations in the multiloculated empyema were reliably dissected under direct vision, and the fibrinopurulent debris and digestive fluids were completely removed. Additionally, thoracoscopy enabled the chest drainage tubes to be placed in suitable sites and the chest lavage could therefore be appropriately performed intra- and postoperatively. Combined thoracoscopic and gastroscopic interventions led to favorable environments both intra- and extraluminally, which facilitated healing and removed the majority of infection within the pleural cavity. This promoted lung re-expansion and improvements in lung function.

Medical thoracoscopy compared with visually-assisted thoracic surgery is minimally invasive and inexpensive. It does not require general anesthesia and double-lumen intubation, which leads to shorter hospitalization times and yields better cosmetic results. Above all, it is a more advisable procedure in frail patients who have high levels of surgical risk $(4,5)$. Although our study presents a small number of patients, the combined application of gastroscopy and thoracoscopy for the treatment of intrathoracic anastomotic leak may be an effective, safe and minimally-invasive procedure.

\section{References}

1. Jiang F, Yu MF, Ren BH, et al: Nasogastric placement of sump tube through the leak for the treatment of esophagogastric anastomotic leak after esophagectomy for esophageal carcinoma. J Surg Res 171: 448-451, 2010.

2. Turkyilmaz A, Eroglu A, Aydin Y, et al: The management of esophagogastric anastomotic leak after esophagectomy for esophageal carcinoma. Dis Esophagus 22: 119-126, 2009.

3. Junemann-Ramirez M, Awan MY, Khan ZM, et al: Anastomotic leakage for esophageal post-esophagogastrectomy carcinoma: retrospective analysis of predictive factors, management and influence on long-term survival in a high volume centre. Eur J Cardiothorac Surg 27: 3-7, 2005.

4. Tassi GF, Davies RJ and Noppen M: Advanced techniques in medical thoracoscopy. Eur Respir J 28: 1051-1059, 2006.

5. Kern L, Robert J and Brutsche M: Management of parapneumonic effusion and empyema: medical thoracoscopy and surgical approach. Respiration 82: 193-196, 2011. 